\title{
Urinary Bladder Cavernous Hemangioma in a 3-year-old: A rare
} case report.

Charles Odongo ${ }^{1}$, raymond atwine ${ }^{1}$, Martin Situma ${ }^{1}$, ambrose okello ${ }^{1}$, eugene ogwang ${ }^{1}$, moses acan ${ }^{1}$, Felix Bongomin ${ }^{2}$, and fred Kirya ${ }^{3}$

${ }^{1}$ Mbarara University of Science and Technology

${ }^{2}$ Gulu University Faculty of Medicine

${ }^{3}$ Soroti University

December 13, 2021

\begin{abstract}
Introduction: Cavernous hemangioma accounts for $0.6 \%$ of bladder tumors. We present a rare case Case Presentation: A 3 -year-old girl presented with intravaginal swelling, dysuria, and hematuria. She received 26 cycles of VAC for embryonal rhabdomyosarcoma. Histopathology confirmed $\mathrm{CH}$. Conclusion: $\mathrm{CH}$ should be considered in the differentials of childhood genitourinary masses.
\end{abstract}

Urinary Bladder Cavernous Hemangioma in a 3-year-old: A rare case report

Charles Newton Odongo ${ }^{1 *}$, Raymond Atwiine ${ }^{1,}$ Martin Situma ${ }^{1}$, Patrick Ambrose Okello ${ }^{1}$, Eugene Ogwang ${ }^{1}$, Moses Achan ${ }^{1}$, Felix Bongomin ${ }^{2}$, Fred Kirya ${ }^{3}$

\section{Affiliations}

Corresponding author

\section{Charles Newton Odongo}

Department of Surgery, Faculty of Medicine, Mbarara University of Science and Technology, Mbarara, Uganda

Mobile: +256774849032

Email:cnodongo@gmail.com

${ }^{1}$ Faculty of Medicine, Mbarara University of Science and Technology, Mbarara, Uganda

${ }^{2}$ Departments of Medical Microbiology \& Immunology, and Internal Medicine, Faculty of Medicine, Gulu University, Gulu, Uganda

${ }^{3}$ Faculty of Medicine, Soroti University, Soroti, Uganda

\section{ABSTRACT}

Introduction: Cavernous hemangioma $(\mathrm{CH})$ of urinary bladder occurs relatively infrequently, accounting for $0.6 \%$ of all bladder tumors. This tumor may occur sporadically or coexist with other benign and malignant vascular lesions. In this report, we present a rare case of $\mathrm{CH}$ in a 3-year-old Ugandan girl.

Case Presentation: A 3-year-old girl was referred to Mbarara Regional Referral Hospital (MRRH) for urological evaluation following a 3-year history of intravaginal swelling, dysuria, and heavy hematuria resulting 
into anemia. Imaging was consistent with polypoid bladder mass arising from the bladder trigone. Embryonal rhabdomyosarcoma was suspected based on clinical eyeballing. She was worked up for chemotherapy and received 26 cycles of vincristine sulfate, actinomycin-d, and cyclophosphamide (VAC). Biopsy and fulguration were performed after optimizing the patient. Histopathology confirmed CH. Surgery was uneventful and resulted to complete cure.

Conclusion: $\mathrm{CH}$ should be considered in the differential diagnosis of childhood genitourinary masses. It is a rare entity in the real-life clinical practice and therefore can be overlooked. Excision biopsy and histology should be performed before initiating the patients to chemotherapy. $\mathrm{CH}$ is very insensitive to chemotherapy and therefore surgery maybe adequate in resource-limited settings.

Key words: Cavernous Hemangioma, Embryonal Rhabdomyosarcoma.

INTRODUCTION: CH of urinary bladder occurs relatively infrequently, accounting for $0.6 \%$ of all bladder tumors (2-4). Bladder hemangiomas result from embryological remains of unipotent angioblastic cells that develop in an anomalous way inside the blood vessels(5). This tumor may occur sporadically or coexist with other benign and malignant vascular lesions such as cutaneous hemangiomas, Klippel-Trenaunay syndrome, Sturge-Weber syndrome, encephalo-trigeminal-angiomatosis, Osler-Weber-Rendu-disease, hemorrhagic telangiectasia syndrome and systemic angiomatosis $(1-3,5)$. CH should be considered in the differential diagnosis of childhood genitourinary masses. It is a rare entity in the real-life clinical practice and therefore can easily be missed. Here, we describe a case of urinary bladder $\mathrm{CH}$ in a 3-year-old female Ugandan.

\section{CASE PRESENTATION}

Presenting symptoms : A 3-year-old girl was referred to MRRH for urological evaluation following a 3 -year history of intravaginal swelling, dysuria, and heavy hematuria resulting into recurrent anemia. There was a history of recurrent blood transfusion, six times before referral to our facility.

Past Medical History: Occasionally treated for febrile illness that the mother attributed to malaria though there were no medical reports to confirm. Mother had visited traditional healer's care before reporting to several health facilities.

Social history: Fifth born of six children, last born died of anemia (malaria), the rest of the siblings are thriving normally. First, second and third born are in primary education. Both the children and mother get support from the father who has a second wife. No history of cancer or hematuria in the first- and second-degree relatives.

Physical examinations: Severe pallor of the conjunctivae, febrile with a temperature of 39.6 degree Celsius. There was no scleral icterus. Local examinations revealed pendulous mass, arising from the urethral meatus held with a stalk and easily bleeds on touching (figure 1A ). Per abdomen: Mild to moderate suprapubic distention and tenderness, dull on percussion.

\section{INVESTIGATIONS}

\section{Table 1: Blood work up and Urinalysis}

\begin{tabular}{llll}
\hline Parameters & $\begin{array}{l}\text { Laboratory values at } \\
\text { admission. }\end{array}$ & $\begin{array}{l}\text { Laboratory values in } \\
\text { the last follow up }\end{array}$ & Reference point \\
\hline $\begin{array}{l}\text { Hemoglobin } \mathrm{g} / \mathrm{dl} \\
\text { Hematocrit }(\%)\end{array}$ & 4.6 & 11.2 & $(9.5-13.5)$ \\
$\begin{array}{l}\text { White cell } \\
\text { counts }\left[10^{\wedge} 3 / u L\right]\end{array}$ & 24.2 & 36 & $(35-44)$ \\
Neutrophils & $34.48^{*} 10^{3}$ & $8.4^{*} 10^{3}$ & $(5.50-17.00)$ \\
Platelets & $18.2^{*} 10^{3}$ & & \\
& $375^{*} 10^{3}$ & $4.6^{*} 10^{3}$ & $(1.50-7.00)$ \\
& & $340^{*} 10^{3}$ & $(150-400)$
\end{tabular}




\begin{tabular}{|c|c|c|c|}
\hline Parameters & $\begin{array}{l}\text { Laboratory values at } \\
\text { admission. }\end{array}$ & $\begin{array}{l}\text { Laboratory values in } \\
\text { the last follow up }\end{array}$ & Reference point \\
\hline creatinine $(\mathrm{mg} / \mathrm{dl})$ & 0.9 & Not repeated & $\begin{array}{l}\text { (0.6-1.1) Females } \\
(0.7-1.3) \text { Males }\end{array}$ \\
\hline $\begin{array}{l}\text { Alanine } \\
\text { Transaminase }(\mathrm{u} / \mathrm{l})\end{array}$ & 12 & Not repeated & $(0-42)$ \\
\hline $\begin{array}{l}\text { Aspartate } \\
\text { Transaminase(u/l) }\end{array}$ & 17 & Not repeated & $(0-37)$ \\
\hline Sodium $(\mathrm{mmol} / \mathrm{l})$ & 129 & Not repeated & $(135-145) \mathrm{mmol} / \mathrm{l}$ \\
\hline Potassium (mmol/l) & 4.1 & Not repeated & $(3.5-5.5) \mathrm{mmol} / \mathrm{l}$ \\
\hline Calcium(mmol/l) & 107 & Not repeated & $(1.1-3.35) \mathrm{mmol} / \mathrm{l}$ \\
\hline Chloride (mmol/l) & 91 & Not repeated & $(95-105) \mathrm{mmol} / \mathrm{l}$ \\
\hline Urinalysis & Urinalysis & Urinalysis & Urinalysis \\
\hline Red blood cells & $\begin{array}{l}>100 \text { red blood } \\
\text { cells/high power field }\end{array}$ & Negative & \\
\hline Leukocytes & $\begin{array}{l}>10 \text { white blood cells/ } \\
\text { high field }\end{array}$ & Negative & \\
\hline Protein & Proteinuria & Negative & \\
\hline
\end{tabular}

Blood work up and Urinalysis : There was severe urosepsis, demonstrated by anemia of $4.6 \mathrm{~g} / \mathrm{dl}$, neutrophilic leukocytosis ( of $34.48^{*} 10^{3} / u L$ ) and thrombocytosis (of $375^{*} 10^{3} / u L$ ). Significantly in urinalysis was haemoglobinuria, proteinuria and leukocytes of more than 10 white blood cells/high field(Table 1 ), these all pointing to urosepsis.

Ultrasound Scan : Pelvic ultrasound scan showed mildly thickened bladder walls with mobile heterogeneously echogenic solid mass seen in the urinary bladder lumen measuring $(5.17 \times 4.10 \mathrm{x} 4.4 \mathrm{~cm})$ with a volume of 49.2cc (figure 2A). The left kidney demonstrated moderately dilated fluid-filled calyces, renal pelvis and proximal ureters (figure $2 \mathrm{~B})$. No focal masses or nephrolithiasis was seen. Right kidney $(7.15 \times 2.83) \mathrm{cm}$ and left kidney $(8.03 \times 3.81) \mathrm{cm}$. There was no prominent trabeculation to suggest cystitis. The uterus and adnexae were normal, free of masses and fluid collections.

Computed Tomography (CT): CT showed a large lobulated polypoid intravesical heterogeneously enhancing mass, appeared to be rising from urinary bladder trigone measuring $5.22 \mathrm{X} 4.80 \mathrm{x} 7.13 \mathrm{~cm}$ in its widest dimensions (figure 3A and figure 3B). Axial and coronal CECT images showed grossly dilated left ureter, pelvis and superior calyx (figure 4A and figure $4 B$ ). There was obliteration of space of Retzius, vesicouterine, rectouterine, vesicovaginal and rectovaginal spaces due to mass effect.

\section{TREATEMENTS}

Medication and Chemotherapy: Antibiotics (metronidazole and ceftriaxone) were initiated for two weeks. The child also received three units of whole blood before initiating on VAC which she received 26 cycles.

Surgery and postoperative treatment: Examination under anesthesia (figure 1B), Excision and Fulguration of the mass was performed under general anesthesia. Child was given general anesthesia and positioned in a lithotomy position and in aseptic technique draped. Urethral meatus was visualized and catheterized with Foleys catheter size 8 Fr (drained bloody urine). We found a foul-smelling polypoid mass protruding from the urethra, highly friable with areas of necrosis (figure 1B). Excision of the mass at the stalk was performed with monopolar diathermy taking care and sparing the urethra. Patient received another unit of whole blood intraoperative. Patient continued ceftriaxone and intravenous paracetamol for the next one week. Postoperative period was uneventful. She was discharge after one week following surgery on oral antibiotics and hematinic as the histology report was to follow. 
Follow up : Patient was followed for six months. Postoperative urinary and pelvic ultrasonography was normal in the last month of follow-ups. Repeated urinalysis was normal. Histopathology (figure 5BA and figure $5 B)$ : Received a single piece of firm dark brown with light brown areas $(1.2 \mathrm{x} 0.8 \mathrm{x} 0.3) \mathrm{cm}$. Sections show extravascular hemorrhage, numerous dilated vascular channels, some of which had thrombosis, and a thin wall. No immature rhabdoblasts consistent with vascular lesion $(\mathrm{CH})$.

\section{DISCUSSION}

The present case highlights a rare presentation of $\mathrm{CH}$ in a 3-year-old masquerading as a primary bladder cancer with recurrent anemia due to heavy hematuria. $\mathrm{CH}$ are benign vascular tumors rarely encountered in the genitourinary tract (1). It is the most common histological form of urinary bladder hemangiomas, with the other forms being capillary and arteriovenous type $(2,3)$. Bladder hemangiomas result from embryological remains of unipotent angioblastic cells that develop in an anomalous way inside the blood vessels (5). Hemangioma of the urinary bladder are rare accounting for $0.6 \%$ of all bladder tumors occurring in all ages, but they are less common during childhood and adolescence (2-4). For children with bladder hemangioma, systemic evaluation is highly recommended because 3-5\% coexist with other genetic syndromes such as cutaneous hemangiomas, Klippel-Trenaunay syndrome, Sturge-Weber syndrome, encephalo-trigeminalangiomatosis, Rendu-Osler-Weber disease, hemorrhagic telangiectasia syndrome and systemic angiomatosis $(1-3,5)$. However, our patient did not have any features suggestive of any of these syndromes.

In the present case, the patient presented with dysuria, hematuria and recurrent anemia which is consistent with case in published literature $(1,5)$. Isolated hematuria results from thrombus, infarction, and angiogenesis by the erosion of the urothelium. Other symptoms include suprapubic pain due to vesicle irritation and urinary retention which may eventually result in obstructive uropathy which was a case for this patient (5). Ultrasonography is the first- line imaging modality for evaluation of pediatric gross hematuria in our setting, as it does not involve ionizing radiation or require sedation, while producing images quickly and painlessly. This is similar to other study (4). Our case showed a mobile heterogeneously echogenic solid mass, with mildly thickened urinary bladder wall at $0.45 \mathrm{~cm}$. Hydroureteronephrosis can occur as a result of ureteric obstruction by the mass, and a hematoma can obscure the mass in the bladder when there is massive bleeding (4). Mild left hydroureteronephrosis was also seen in this case due to mass effect. Computed tomography (CT), pelvic arteriography, and magnetic resonance imaging are useful in defining and location of a hemangioma but are not helpful diagnostic tools in distinguishing bladder hemangiomas from other bladder tumors (3).

In our setting however, we can only perform CT and ultrasonography for any pelvic mass. Cystoscopy usually reveal a reddish-blue, polypoid, submucosal mass with intact urothelium $(3,6)$, however we were not able to perform cystoscopy because of lack of its availability.

Treatment of $\mathrm{CH}$ depends on, size, location, and depth of penetration. For small lesions, and asymptomatic hemangiomas, surveillance is sufficient. The treatment is only necessary when the lesions threaten the organ function or the patients' performance status, such as hematuria, anemia, and suspicion of malignant lesion. Our case developed recurrent anemia, obstructive uropathy and urosepsis (evidence by neutrophilic leukocytosis), justifying surgical approach. Treatment options usually involves observation, transurethral resection, electrocoagulation, radiation, systemic steroids administration, injection of a sclerosing agent, interferon-a-2 therapy, laser therapy, and partial cystectomy or complete cystectomy (3).

In our case, biopsy and fulguration was adequate to treat the lesion. Bladder $\mathrm{CH}$ are histologically similar to hemangiomas found at other sites with numerous proliferative capillaries mixed with thin walled, dilated blood-filled vessels lined with flattened endothelium. The vessels are sometimes thickened by adventitial fibrosis $(1,2)$. Sections for our case show extravascular hemorrhage, numerous dilated vascular channels containing red blood cells (figure 5 ), some of which had thrombosis, and a thin wall. No immature rhabdoblasts.

Conclusion: $\mathrm{CH}$ should be considered in the differential diagnosis of childhood genital embryonal rhabdomyosarcoma. Excision biopsy and histology should be performed before initiating the patients to 
chemotherapy to avoid toxicities. $\mathrm{CH}$ is insensitive to chemotherapy and therefore surgery alone is adequate.

Data sharing statement: The information used and/or analyzed during this case report is available from the corresponding author on reasonable request.

Ethics: Ethical clearance was obtained from Mbarara University Faculty of Medicine Research Committee, MUST-29/06-21. The mother provided an informed written consent for the image of her child and other clinical information to be reported in the journal.

\section{Consent for publication.}

The patient provided an informed written consent for this case to be published in a peer-reviewed journal

Author's contributions : O.C.N performed conceptualization, data acquisition, drafting of the case report and final approval of the version to be published. AR evaluated the pathology of this case. P.A.O, E.O and M.A interpreted radiological imaging, B.F was the main editor of the final case report. F.O Edited the work and S.M supervised surgery during excision and biopsy and approved the final version to be published.

Acknowledgement: Surgical, pathology, pediatric oncology and radiology Departments of Mbarara University and the study participant.

Competing interest : The authors declare no conflict of interest

Financial support: None to declare

\section{REFERENCES}

1. Sajitha K, Prasad HK, Pradeep A, Rajeev T, Mathias M, Shetty KJ. Cavernous hemangioma of ureter masquerading as malignancy-A rare case report. Urological Science. 2020;31(3):139.

2. Syu S-H, Chan K-S, Hsiao C-H, Chen W-Y, Lee L-M, Wen Y-C. A large urinary bladder hemangioma mimicking urachal cancer: a case report and literature review. Urology. 2019;123:224-6.

3. Zhao G, Mishra P, Yang J, Ma T, Deng C, Ke C. Case Report on Hemangioma of The Urinary Bladder: two rare cases and literature review. 2020.

4. Kim YY, Kim M-J, Lee M-J, Kim J-Y. Multiple hemangiomas of the urinary bladder in a child with gross hematuria. Ultrasonography. 2015;34(3):231.

5. Jibhkate S, Sanklecha V, Valand A. Urinary bladder hemangioma-a rare urinary bladder tumor in a child. APSP journal of case reports. 2015;6(1):6.

6. Kim GP, Kwon DD, Oh BR, Park KS, Ryu SB, Park YI. A Case of Cavernous Hemangioma of the Urinary Bladder. Korean Journal of Urology. 1999;40(5):655-8. 

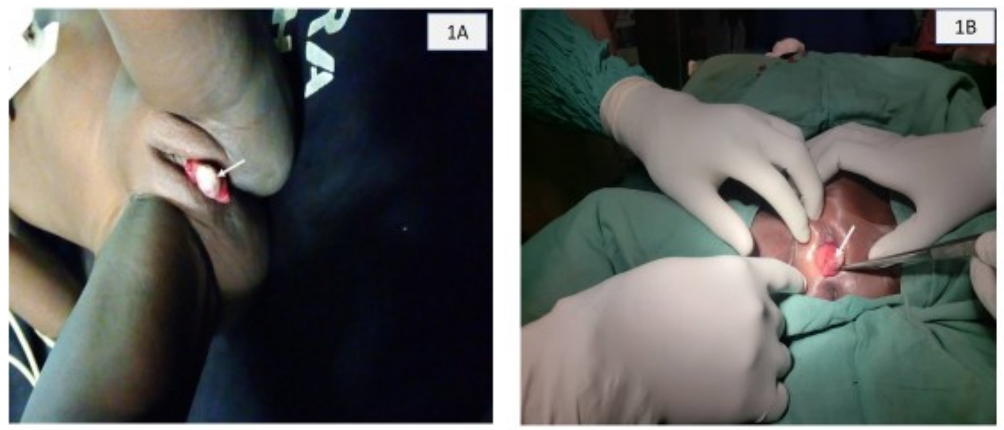

Figure 1:1Apolypoid mass viewed during examination,1B-viewed during examination under anesthesia, excision and fulguration was performed.
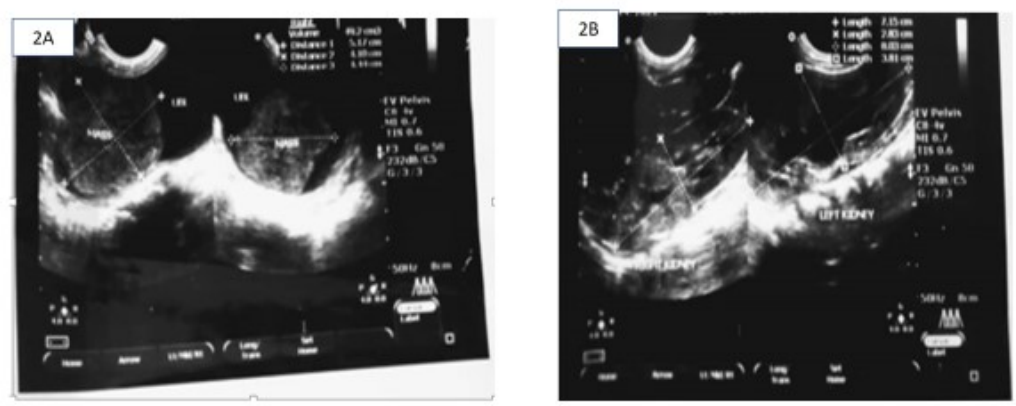

Figure 2:Transverse and longitudinal abdominal ultrasound images show lobulated heterogeneous solid mass in the urinary bladder lumen (A) and moderately dilated left renal calyces(B).
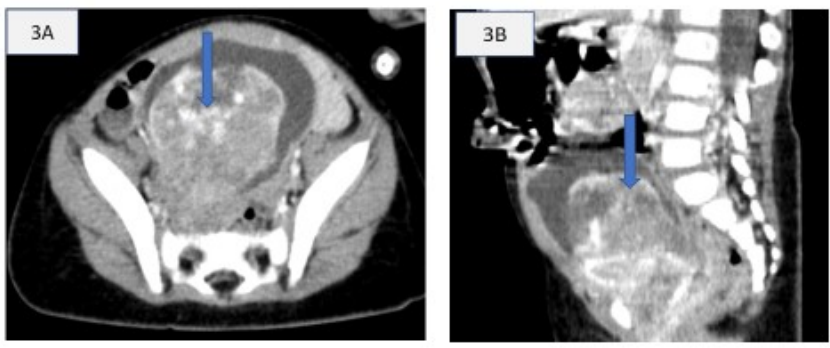

Fig 3 ( $A$ and B) Axial and sagittal CECT images show a large lobulated polypoid intravesical heterogeneously enhancing mass, which appears to arise from the trigone of the urinary bladder(blue arrows) 

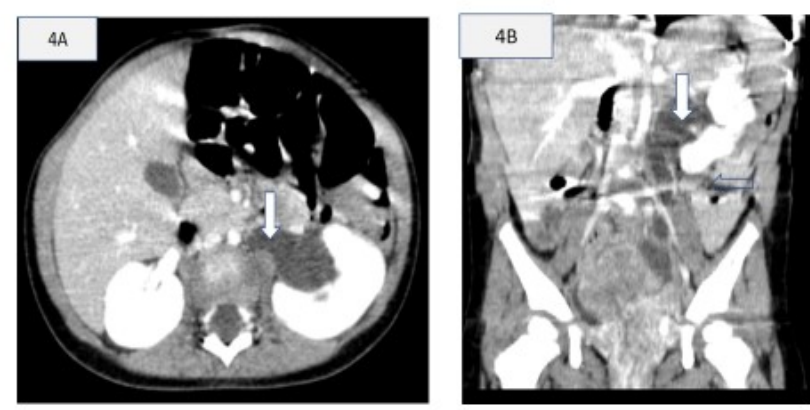

Figure 4: Axial (A) and coronal (B) CECT images show left hydroureteronephrosis (white arrows).
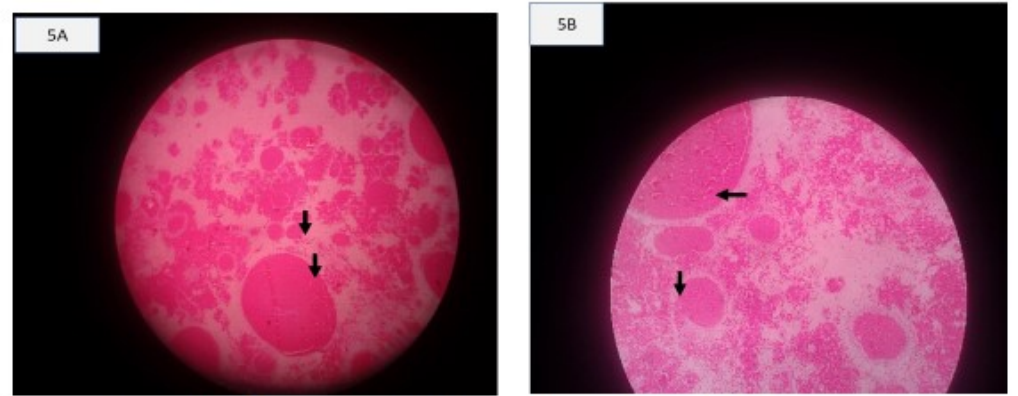

Figure 5 : Numerous dilated vascular channels laden with red blood cells indicated in black arrows in $A$ and $B$ 